1 Contamination of hospital tap water: the survival and persistence of Pseudomonas

2 aeruginosa on conventional and 'antimicrobial' outlet fittings

\title{
3 SUMMARY
}

4 Background: Pseudomonas aeruginosa infections have been linked to contaminated hospital taps,

5 highlighting the potential for tap outlet fittings (OF) to harbour biofilm. P. aeruginosa may be

6 transferred to OFs via contaminated cleaning-cloths. Suggested interventions include flushing

7 regimens and alternative OF designs.

8 Aim: To investigate the transfer of $P$. aeruginosa from a contaminated cleaning-cloth to 9 conventional and 'antimicrobial/anti-biofilm' OFs and to determine whether this contamination 10 persists and/or leads to contamination of tap water. different types of OF (one of conventional design (OF-A) and three marketed as 'antimicrobial' and/or 'anti-biofilm' (OF- B, -C and -D)). OFs were inserted into an experimental water distribution system for up to 24-hours. Survival was assessed by culture. Single and multiple water samples were collected and cultured for $P$. aeruginosa.

Findings: The median number of $P$. aeruginosa transferred from cloth to OF was $5.7 \times 10^{5} \mathrm{CFU}(\mathrm{OF}-\mathrm{A})$, $1.9 \times 10^{6} \mathrm{CFU}(\mathrm{OF}-\mathrm{B}), 1.4 \times 10^{5} \mathrm{CFU}$ (OF-C) and $2.9 \times 10^{6} \mathrm{CFU}$ (OF-D). Numbers declined on all OFs during the 24-hour period with log reductions ranging from 3.5 (OF-C) to 5.2 (OF-B; p>0.05). All water samples delivered immediately after OF contamination contained $P$. aeruginosa at $\geq 10 \mathrm{CFU} / 100 \mathrm{~mL}$. Contamination of water delivered from OF-A persisted despite continued flushing. Water delivered from OF-B did not contain $P$. aeruginosa beyond the first flush.

Conclusion: Contaminated cleaning-cloths can transfer $P$. aeruginosa to OFs, leading to contamination of tap water. Whilst not removing the potential for contamination, 'antimicrobial/anti-biofilm' OFs may prevent $P$. aeruginosa from continually contaminating water delivered from the outlet.

\section{INTRODUCTION}

Water outlets, particularly taps and associated pipework, are a recognised reservoir for microorganisms, providing a large surface area for biofilms to develop and to harbour potential pathogens, such as Pseudomonas aeruginosa ${ }^{1}$. In the UK, $P$. aeruginosa is responsible for $3 \%$ of all reported monospecies bacteraemias ${ }^{2}$. There are also reports of non-bacteraemic infections ${ }^{3,4}$. The infection of immunocompromised individuals by $P$. aeruginosa carries a risk of fatality ${ }^{5}$ but, as reporting is voluntary, the exact incidence and mortality of $P$. aeruginosa infections is unknown. The association between hospital taps and $P$. aeruginosa infections is well documented ${ }^{6,7}$, however, it was not until 2013, that the Department of Health (DoH) issued guidance relating to $P$. aeruginosa control ${ }^{8}$.

P. aeruginosa readily forms biofilms ${ }^{9}$, and the design and/or materials associated with different tap components, such as thermostatic mixing valves $(\mathrm{TMVs})^{10}$, solenoid valves ${ }^{11}$ and outlet fittings $(\mathrm{OFs})^{12}$, have been shown to facilitate biofilm formation. $P$. aeruginosa is able to persist on wet and dry surfaces for extended periods of time ${ }^{11,13}$. 
1 Between November 2011 and January 2012, 25 babies admitted to neonatal intensive care units in

Northern Ireland acquired $P$. aeruginosa. These incidents were attributed to contaminated OFs ${ }^{12}$. However, in a laboratory-based investigation, colonisation of OFs was minimal ${ }^{11}$. An acknowledged limitation of this study was that the tap assemblies were inoculated systemically, simulating contamination of the water supply. Hospital taps, however, may also be exposed to contamination originating from the clinical environment, i.e. retrograde contamination.

Retrograde contamination could occur in a number of different ways, many resulting from human behaviour. OFs can become contaminated via splashback from a contaminated waste trap ${ }^{14}$ or surface $^{15}$ (e.g. while rinsing contaminated equipment) or through inappropriate disposal and/or splashing of contaminated patient fluids ${ }^{16}$. The potential spread of contamination during cleaning has also been demonstrated ${ }^{17}$. Contamination of environmental surfaces via microfibre cloths is well documented ${ }^{18,19}$, and can occur even when using the previously published fold-and-refold method ${ }^{20}$. Data on the potential for microfibre cloths to contaminate OFs has not been published. However, in response to reports of OF contamination and DoH guidance, several manufacturers have engineered a variety of 'antimicrobial' or 'anti-biofilm' solutions, designed to prevent the survival of microorganisms introduced to the OF, and/or to minimise the adhesion of microorganisms and detritus.

The aim of this study was to investigate retrograde contamination, specifically whether conventional and 'antimicrobial/anti-biofilm' OFs can become contaminated with $P$. aeruginosa via cleaning cloths and if this contamination can persist and/or lead to contamination of tap water.

\section{METHODS}

\section{Preparation of test suspension}

Cryopreserved Pseudomonas aeruginosa, previously isolated from an experimental water distribution system (EWDS) ${ }^{11}$, was resuscitated from beads (Technical Service Consultants Ltd., Lancashire, UK) onto Tryptone Soya agar (Oxoid, Basingstoke, UK) and subcultured onto Cetrimide (CN) agar (Oxoid). Liquid cultures were prepared by suspending a colony in $100 \mathrm{~mL}$ of nutrient broth (Oxoid) and incubating at $37^{\circ} \mathrm{C}$ with shaking at $120 \mathrm{rpm}$ for 16 -hours.

\section{Selection of outlet fittings (OFs)}

Four different OFs were selected for this study: one of conventional design (OF-A) and three (currently on the market or in development) designed to reduce the risk of microbial colonisation. In comparison to OF-A, a multi-layered plastic rosette ${ }^{11}$, OF-B is simpler in design and comprises a single-bore with a copper interior lining. OF-C has a similar simplified design but is made from a plastic that the manufacturer claims reduces impurity (and bacterial) adherence. OF-D has a conventional-style flow-straightening device (similar to OF-A) but made from a silver-impregnated plastic. A copper alloy sheath $(>76 \% \mathrm{Cu}$ ) projects beyond this internal device.

\section{Contamination of outlet fittings via microfibre cloth}

Microfibre cleaning cloths ( $80 \%$ polyester and $20 \%$ polyamide; Arco, Hull, UK) were cut into $10 \mathrm{~cm}^{2}$ swatches, weighed and inoculated with $10 \mathrm{~mL}$ of the bacterial test suspension ( $10^{8}$ colony forming units (CFU)/mL). Each swatch was wrung and reweighed, before being used to wipe the accessible 
1 surfaces of between 1 and 9 OFs for 10 seconds. Cloth-contaminated OFs were inserted into taps on

2 the EWDS and left in situ for 15 minutes, one-, four-, eight-, 12- or 24-hours.

\section{Survival of $P$. aeruginosa on outlet fittings}

$4 \quad$ At the appropriate time point, OFs $(n=3)$ were removed from the taps and transferred to $5 \mathrm{~mL}$ sterile 5 thiosulphate Ringers solution (Oxoid) or, to (when it was required) neutralise potential antimicrobial 6 ions, $5 \mathrm{~mL}$ Dey-Engley neutralising broth (Sigma-Aldrich, Dorset, UK). Each OF was vortexed with 7 sterile glass beads for two minutes and the resulting suspension serially diluted (ten-fold). Aliquots $8(100 \mu \mathrm{L})$ of each dilution were plated onto $\mathrm{CN}$ agar and incubated at $37^{\circ} \mathrm{C}$ for $24-48$ hours. $3 \mathrm{~mL}$ of 9 the remaining suspension was filtered through a $0.2 \mu \mathrm{m}$ membrane (Pall Life Sciences, Portsmouth, 10 UK) which was transferred to $\mathrm{CN}$ agar and incubated at $37^{\circ} \mathrm{C}$ for $24-48$ hours. Each experiment was 11 repeated on four separate occasions.

\section{Detection of $P$. aeruginosa in water dispensed from contaminated outlet fittings}

OFs (contaminated as previously described) were inserted into the EWDS. Each tap was flushed (30seconds, $4.5 \mathrm{~L} / \mathrm{min}$ ) either once over a 24-hour period (OF-A only), or five times over a 20-minute period to simulate an infrequently- or frequently-used tap respectively (all OF types). A TMV associated with each tap ensured the temperature of the water delivered from each outlet was $\sim 43^{\circ} \mathrm{C}$. Chlorine levels were approximately $0.03-0.22 \mathrm{mg} / \mathrm{L}$. During each flush, water samples $(500 \mathrm{~mL})$ were collected in sample bottles containing $20 \mathrm{mg} / \mathrm{L}$ sodium thiosulphate (Scientific Laboratory Supplies, Nottingham, UK). The presence of $P$. aeruginosa was determined by filtering $100 \mathrm{~mL}$ of each water sample through a $0.2 \mu \mathrm{m}$ membrane. Membranes were transferred to $\mathrm{CN}$ agar and incubated at $37^{\circ} \mathrm{C}$ for $24-48$ hours. Colonies were enumerated and results recorded as above ( $\geq 10 \mathrm{CFU} / 100 \mathrm{~mL}$ ) or below $(1-9 \mathrm{CFU} / 100 \mathrm{~mL})$ the hospital alert limit for augmented care ${ }^{8}$, or below the detection limit of the assay (<1CFU/100mL). Experiments were repeated on four separate occasions.

\section{Data analysis}

Data analysis was performed using GraphPad Prism (version 7.00 for Windows). Parametric data

26 were compared using unpaired T-tests or one-way ANOVA followed by Šídák's multiple comparisons test. Non-parametric data were compared using Kruskal-Wallis one-way ANOVA in combination with Dunn's multiple comparisons test. Statistical significance was set at $p<0.05$. Chi-square $\left(X^{2}\right)$ tests were performed to test for independence between contamination levels of flushed water and the number of flushes.

\section{RESULTS}

\section{Contamination of outlet fittings via contaminated microfibre cloths}

$P$. aeruginosa was transferred to all OFs via contaminated microfibre cloths. The median number ( $n=12$ ) of $P$. aeruginosa transferred was $5.7 \times 10^{5} \mathrm{CFU}$ (OF-A), $1.9 \times 10^{6} \mathrm{CFU}$ (OF-B), $1.4 \times 10^{5} \mathrm{CFU}$ (OF-C) and $2.9 \times 10^{6} \mathrm{CFU}(\mathrm{OF}-\mathrm{D})$. Significantly fewer bacteria were transferred to OF-C than to any other OF

\section{Survival of $P$. aeruginosa over time}


1 Figure 1 illustrates the reduction in viable $P$. aeruginosa recovered from the different OFs up to 24-

hours after contamination. For all OF types, significant $\log _{(10)}$ reductions were observed by $15-$ minutes $(p<0.05)$. There were also significant $\log _{(10)}$ reductions between one- and four-hours for all OF types, with reductions of 2.7 (OF-A), 3.7 (OF-B), 2.2 (OF-C) and 2.6 (OF-D). The rate of $P$. aeruginosa reduction decreased after the first four-hours, with significant $\log _{(10)}$ reductions of 1.8 (OF-A), 1.6 (OF-B), 1.3 (OF-C) and 2.6 (OF-D) over the following 20-hour period. No significant reduction was seen between four and eight-hours ( $p>0.05)$ and the subsequent four-hour period saw variable trends across the OF designs, with only OF-B demonstrating a significant $\log _{(10)}$ reduction of 1.1 .

No significant difference in $P$. aeruginosa viability was observed after 24-hours between the conventional and 'antimicrobial/anti-biofilm' OFs. OF-B showed greater $\log _{(10)}$ reduction than OF-A at 12 -hours $(p<0.05)$, however this did not carry through to the 24-hour time point.

\section{Detection of $\boldsymbol{P}$. aeruginosa in water dispensed from contaminated outlet fittings}

Taps fitted with OF-A were flushed once to simulate an infrequently-used tap. All water samples $(n=6)$ taken immediately after contamination of the OF were positive for $P$. aeruginosa at $\geq 10$ CFU/100mL (above the augmented care alert limit). The level of $P$. aeruginosa in all water collected at 15-minutes, one-, four- and 12-hours after contamination also exceeded the alert limit. $P$. aeruginosa persisted on the surface of the OF and 24-hours after contamination, 3/6 (50\%) of water samples delivered through OF-A contained $P$. aeruginosa at levels $\geq 10 \mathrm{CFU} / 100 \mathrm{~mL}$.

Regardless of OF design, increased usage of the tap correlated with reduced water contamination (Table I). In all cases, water delivered immediately after contamination of the OFs (flush 1 ) was positive for $P$. aeruginosa at $\geq 10 C F U / 100 \mathrm{~mL}$. Contamination of water delivered from OF-A persisted despite continued flushing (or usage) and 6/12 (50\%) in the fifth flush contained $P$. aeruginosa at levels of $\geq 10 \mathrm{CFU} / 100 \mathrm{~mL}$ (Table I). In contrast, by the second flush, the level of $P$. aeruginosa in water delivered through OF-B was always below the detection limit of the assay. Whilst two (17\%) and one $(8 \%)$ of the 12 water samples collected during the second and third use of taps fitted with OF-C respectively contained $P$. aeruginosa at $\geq 10$ CFU/100mL, the majority of samples $(11 / 12,92 \%)$ were below detection by the fourth flush. $P$. aeruginosa was recovered from water delivered from OF-D at $\geq 10 C F U / 100 \mathrm{~mL}$ until the fourth flush, by which point no $P$. aeruginosa was detected in $8 / 12$ (67\%) samples. In contrast to OF-B and -C, water dispensed by contaminated OF-D contained $P$. aeruginosa at detectable levels in $2 / 12$ (17\%) samples by the fifth flush. $X^{2}$ tests indicated that the level of contamination was not independent from the number of flushes that had occurred for all four OF types (i.e. there is an association between flushing and the observed reduction in $P$. aeruginosa counts) ( $\mathrm{n}=12$. OF-A: $X^{2}(4)=10.7 p<0.05$; OF-B $X^{2}(4)=60.0 p<0.0001$; OF-C: $X^{2}(4)=$ 46.2, $p<0.0001$; OF-D $\left.X^{2}(4)=49.3, p<0.0001\right)$.

\section{DISCUSSION}

Although the colonisation of OFs with $P$. aeruginosa has been reported since the $1960 \mathrm{~s}^{21}$, very few studies have investigated how such contamination can occur. Of those that have, the focus has been on post-incident investigation ${ }^{12}$ or systemic contamination ${ }^{11}$. This is the first study to investigate the 
1 transfer of $P$. aeruginosa from a cloth to an OF (i.e. cross-contamination during cleaning). The high load of $P$. aeruginosa used to inoculate the microfibre cloths was comparable to contamination levels found in cleaning-cloths in previous studies ${ }^{22}$ and resulted in OF contamination at levels comparable to that recovered from the OFs implicated in the Northern Ireland incidents $\left(1.8 \times 10^{5} \mathrm{CFU}\right)^{12}$. P. aeruginosa can survive on dry surfaces ${ }^{13}$ and in the absence of flushing, $P$. aeruginosa survived on the surface of all OFs for at least 24 hours.

The antimicrobial effect of copper and silver ions is well documented and such studies have led to the design and manufacture of OFs that incorporate 'antimicrobial' materials ${ }^{23,24}$. However, results from laboratory-based assays do not necessarily translate when applied ${ }^{25}$. Copper surfaces can, in laboratory studies, achieve a 6 - $\log _{(10)}$ reduction in bacterial viability ${ }^{26}$. In contrast, within the healthcare setting, copper surfaces have been shown to reduce the level of environmental contamination by just $\sim 2-\log _{(10)} 27,28$, and despite microbial reductions being significantly greater than on control surfaces, copper surfaces are still contaminated at levels above the accepted limit for healthcare $\left(2.5 \mathrm{CFU} / \mathrm{cm}^{2}\right)^{29}$. The results of the current study demonstrate that OFs can become contaminated when wiped with a contaminated cloth, and this contamination can persist. In comparison to the conventional OF (OF-A), the OFs incorporating antimicrobial materials including copper (i.e. OF-B and-D) did not significantly reduce microbial contamination over a 24-hour period. The clinical tap industry is increasingly incorporating copper and brass components within new designs whilst also attempting to reduce surface roughness to minimise niches for bacterial attachment. However, a study by Zeiger et al highlighted the importance of surface roughness in the efficiency of copper contact killing, demonstrating a superior killing effect by electroporated copper compared to polished or deposited copper surfaces ${ }^{30}$.

The transfer of $P$. aeruginosa to OFs led to contamination of the first sample of water delivered from all outlets at $\geq 10 \mathrm{CFU} / 100 \mathrm{~mL}$. Should the tap be used less frequently (i.e. once within a 24 -hour period) then the ability of $P$. aeruginosa to persist on the surface of conventional OFs could result in 'high-risk' water (containing $\geq 10 \mathrm{CFU} / 100 \mathrm{~mL}^{8}$ ) being delivered 24-hours after the contamination event. Regardless of OF design, $P$. aeruginosa levels in water dispensed from affected taps reduced with increased tap usage. The 'antimicrobial/anti-biofilm' OFs reduced contamination more efficiently than conventional OFs over a series of flushes. The contamination of water delivered by OF-B and OF-C was reduced to a level below the detection limit of the assay ( $<1 \mathrm{CFU} / 100 \mathrm{~mL}$ ) after one 30-second flush. However, unlike OF-B, OF-C did not consistently clear residual contamination, meaning that water samples delivered during the second (2/12) and third flush (1/12) were contaminated at $\geq 10 \mathrm{CFU} / 100 \mathrm{~mL}$. OF-B and $-\mathrm{C}$ are both single bore OFs. OF-D is more complex and was less efficient than OF-B and $-C$ at clearing contamination. This could be due to the additional flow control provided by its internal device, which is similar to OF-A. It is possible that antimicrobial agents from OF-D acted upon $P$. aeruginosa in the water sample, hence its superior performance to OF-A.

When wiped with a contaminated microfibre cloth, significantly fewer bacteria were transferred to OF-C than to any of the other OFs, which, when considered alongside the rapid removal of contamination through tap usage, implies that $P$. aeruginosa is unable to attach as easily or as strongly to this OF design. OF-C is marketed as being 'anti-biofilm'. However, it is unclear whether clearance of $P$. aeruginos $a$ was an artefact of being retrofitted into another manufacturer's tap, or due to the materials used. Not all OFs are able to maintain flow regulation performance across all 
spout designs. It is feasible that turbidity of flow and the resulting shear forces may enhance sloughing of attached bacteria. Nonetheless, as was observed during the current investigation, poor flow regulation can lead to splashing (data not presented). Implications of water (and its microbial content) spreading to the surrounding environment must be taken into consideration, both in droplet and aerosol form ${ }^{31}$.

This study has demonstrated that OFs could become contaminated with $P$. aeruginosa in a retrograde manner, for example, during cleaning. If not immediately removed, this contamination can persist and lead to contamination of tap water. Whilst not removing the potential for retrograde contamination, 'antimicrobial/anti-biofilm' OFs may, in combination with a flushing regimen, prevent $P$. aeruginosa from continually contaminating the water delivered from the outlet. Retrofitting taps with 'antimicrobial/anti-biofilm' OFs is possible but this may not be easy or appropriate. Some designs would require the replacement of the entire tap assembly, whilst the simplification of other designs has had a negative impact upon the original function of the OFs (i.e. to regulate and straighten the flow of water).

Furthermore, relying on the antimicrobial properties of material (or OF) is an insufficient infection control measure. Flushing and frequent tap use reduces water system stagnation allowing any decontamination agents added to the tank water to reach the tap outlet and OF. It is important to remember that augmented care units, where taps tend to be used very frequently ${ }^{32}$, are not the only wards that immunocompromised patients spend time on, and $P$. aeruginosa infections have been acquired from outpatients clinics ${ }^{33}$. Immunocompromised outpatients receiving prolonged treatment, such as oncology and cystic fibrosis patients, visit day wards and clinics regularly, and also make unexpected presentations to emergency departments ${ }^{34}$. Retrograde contamination of sinks, taps and OFs could occur regardless of ward. It is, therefore, important to maintain and flush all hospital taps regularly and essential that factors contributing to retrograde contamination (be it human behaviour or otherwise) be investigated and addressed.

\section{ACKNOWLEGDEMENTS}

The authors would like to thank participating manufacturers for supplying the alternative outlet fittings.

\section{Conflict of interest statement}

The views expressed in this publication are those of the authors and not of Public Health England.

\section{Funding sources}

Healthcare Infection Society

\section{REFERENCES}

1 September, S. M., Els, F. A., Venter, S. N. \& Brözel, V. S. Prevalence of bacterial pathogens in biofilms of drinking water distribution systems. Journal of water and health 5, 219-227, (2007).

2 Public Health England. Surveillance of Pseudomonas and Stenotrophomonas species causing bacteraemia in England, Wales and Northern Ireland: 2014. (2015). 
3 Bjarnsholt, T. et al. Pseudomonas aeruginosa biofilms in the respiratory tract of cystic fibrosis patients. Pediatric pulmonology 44, 547-558, (2009).

4 Estahbanati, H. K., Kashani, P. P. \& Ghanaatpisheh, F. Frequency of Pseudomonas aeruginosa serotypes in burn wound infections and their resistance to antibiotics. Burns 28, 340-348, (2002).

$5 \quad$ Kang, C.-I. et al. Pseudomonas aeruginosa bacteremia: risk factors for mortality and influence of delayed receipt of effective antimicrobial therapy on clinical outcome. Clinical Infectious Diseases 37, 745-751, (2003).

6 Ferroni, A. et al. Outbreak of nosocomial urinary tract infections due to Pseudomonas aeruginosa in a paediatric surgical unit associated with tap-water contamination. Journal of Hospital Infection 39, 301-307, (1998).

7 Rogues, A. M. et al. Contribution of tap water to patient colonisation with Pseudomonas aeruginosa in a medical intensive care unit. Journal of Hospital Infection 67, 72-78, (2007).

8 Department of Health. HTM 04-01 - Addendum: Pseudomonas aeruginosa-advice foraugmented care units. (2013).

9 Sauer, K., Camper, A. K., Ehrlich, G. D., Costerton, J. W. \& Davies, D. G. Pseudomonas aeruginosa displays multiple phenotypes during development as a biofilm. Journal of Bacteriology 184, 1140-1154, (2002).

10 Quick, J. et al. Seeking the source of Pseudomonas aeruginosa infections in a recently opened hospital: an observational study using whole-genome sequencing. BMJ open 4, e006278, (2014).

11 Moore, G. et al. Biofilm formation in an experimental water distribution system: the contamination of non-touch sensor taps and the implication for healthcare. Biofouling 31, 677-687, (2015).

12 Walker, J. et al. Investigation of healthcare-acquired infections associated with Pseudomonas aeruginosa biofilms in taps in neonatal units in Northern Ireland. Journal of Hospital Infection 86, 16-23, (2014).

13 Kramer, A., Schwebke, I. \& Kampf, G. How long do nosocomial pathogens persist on inanimate surfaces? A systematic review. BMC Infectious Diseases 6, 130, (2006).

14 Zhou, Z. et al. Sources of sporadic Pseudomonas aeruginosa colonizations/infections in surgical ICUs: Association with contaminated sink trap. Journal of Infection and Chemotherapy 22, 450-455, (2016).

15 Hota, S. et al. Outbreak of Multidrug Resistant Pseudomonas aeruginosa Colonization and Infection Secondary to Imperfect Intensive Care Unit Room Design. Infection Control and Hospital Epidemiology 30, 25-33, (2009).

16 Balm, M. et al. Bad design, bad practices, bad bugs: frustrations in controlling an outbreak of Elizabethkingia meningoseptica in intensive care units. Journal of Hospital Infection 85, 134140, (2013).

17 Garvey, M. I., Bradley, C. R. \& Bradley, C. W. Evaluating the risks of wash hand basin tap disinfection. Journal of Hospital Infection 94, 21-22.

18 Moore, G. \& Griffith, C. A laboratory evaluation of the decontamination properties of microfibre cloths. Journal of Hospital Infection 64, 379-385, (2006).

19 Scott, E. \& Bloomfield, S. F. The survival and transfer of microbial contamination via cloths, hands and utensils. Journal of Applied Bacteriology 68, 271-278, (1990).

20 Bergen, L. K., Meyer, M., Høg, M., Rubenhagen, B. \& Andersen, L. P. Spread of bacteria on surfaces when cleaning with microfibre cloths. Journal of Hospital Infection 71, 132-137, (2009).

21 Wilson, M. G., Nelson, R. C., Phillips, L. H. \& Boak, R. A. New source of Pseudomonas aeruginosa in a nursery. Journal of the American Medical Association 175, 1146-1148, (1961). 
22 Yepiz-Gomez, M. S., Bright, K. R. \& Gerba, C. P. Identity and numbers of bacteria present on

2

3

4

5

6

7

8

9

10

11

12

13

14

15

15 tabletops and in dishcloths used to wipe down tabletops in public restaurants and bars. Food Protection Trends 26, 786-792, (2006).

23 Eduardo, J. F. et al. The preparation of highly active antimicrobial silver nanoparticles by an organometallic approach. Nanotechnology 19, 185602, (2008).

24 Arijit Kumar, C., Ruchira, C. \& Tarakdas, B. Mechanism of antibacterial activity of copper nanoparticles. Nanotechnology 25, 135101, (2014).

25 Mikolay, A. et al. Survival of bacteria on metallic copper surfaces in a hospital trial. Applied Microbiology and Biotechnology 87, 1875-1879, (2010).

26 Warnes, S. L., Green, S. M., Michels, H. T. \& Keevil, C. W. Biocidal efficacy of copper alloys against pathogenic enterococci involves degradation of genomic and plasmid DNAs. Applied and Environmental Microbiology 76, 5390-5401, (2010).

27 Schmidt, M. G. et al. Sustained reduction of microbial burden on common hospital surfaces through introduction of copper. Journal of Clinical Microbiology 50, 2217-2223, (2012).

28 Rai, S. et al. Evaluation of the antimicrobial properties of copper surfaces in an outpatient infectious disease practice. Infection Control \& Hospital Epidemiology 33, 200-201, (2012).

29 Dancer, S. J. How do we assess hospital cleaning? A proposal for microbiological standards for surface hygiene in hospitals. Journal of Hospital Infection 56, 10-15, (2004).

30 Zeiger, M., Solioz, M., Edongué, H., Arzt, E. \& Schneider, A. S. Surface structure influences contact killing of bacteria by copper. MicrobiologyOpen 3, 327-332, (2014).

31 Beggs, C. The airborne transmission of infection in hospital buildings: fact or fiction? Indoor and Built Environment 12, 9-18, (2003).

32 Panhotra, B., Saxena, A. \& Al-Arabi, A.-G. A. The effect of a continuous educational program on handwashing compliance among healthcare workers in an intensive care unit. British Journal of Infection Control 5, 15-18, (2004).

33 Tümmler, B. et al. Nosocomial acquisition of Pseudomonas aeruginosa by cystic fibrosis patients. Journal of Clinical Microbiology 29, 1265-1267, (1991).

34 McKenzie, H. et al. Chemotherapy outpatients' unplanned presentations to hospital: a retrospective study. Supportive Care in Cancer 19, 963-969, (2011). 
1

1 Figures and Legends:

2 Table I The number of water samples delivered through contaminated outlet fittings that contained $3 P$. aeruginosa at levels above $(\geq 10 \mathrm{CFU} / 100 \mathrm{~mL})$ or below $(1-9 \mathrm{CFU} / 100 \mathrm{~mL})$ the hospital alert limit for 4 augmented care, or below the detection limit of the assay ( $<1 \mathrm{CFU} / 100 \mathrm{~mL})$. Samples $(n=12)$ were 5 collected over five consecutive tap usages (i.e. five consecutive 30 second flushes).

6 Figure 1. Reduction $\left(\log _{(10)}\right)$ in cloth-transferred $P$. aeruginosa on outlet fitting types [mean $(n=12)$ $7 \quad \pm$ standard deviation] 
Table I The number of water samples delivered through contaminated outlet fittings that contained $P$. aeruginosa at levels above $(\geq 10 \mathrm{CFU} / 100 \mathrm{~mL}$ ) or below $(1-9 \mathrm{CFU} / 100 \mathrm{~mL})$ the hospital alert limit for augmented care, or below the detection limit of the assay $(<1 \mathrm{CFU} / 100 \mathrm{~mL})$. Samples $(n=12)$ were collected over five consecutive tap usages (i.e. five consecutive 30 second flushes).

\section{OF type $P$. aeruginosa Frequency of tap use (CFU/100mL)}

\begin{tabular}{lcrrrrr}
\hline & & $\mathbf{1}^{\text {st }}$ flush & $\mathbf{2}^{\text {nd }}$ flush & $\mathbf{3}^{\text {rd }}$ flush & $\mathbf{4}^{\text {th }}$ flush & $\mathbf{5}^{\text {th }}$ flush \\
\hline OF-A & $\geq 10$ & 12 & 11 & 8 & 8 & 6 \\
& $1-9$ & 0 & 1 & 2 & 1 & 2 \\
& $<1$ & 0 & 0 & 2 & 3 & 4 \\
\hline OF-B & $\geq 10$ & 12 & 0 & 0 & 0 & 0 \\
& $1-9$ & 0 & 0 & 0 & 0 & 0 \\
& $<1$ & 0 & 12 & 12 & 12 & 12 \\
\hline OF-C & $\geq 10$ & 12 & 2 & 1 & 0 & 0 \\
& $1-9$ & 0 & 0 & 0 & 1 & 0 \\
& $<1$ & 0 & 10 & 11 & 11 & 12 \\
\hline OF-D & $\geq 10$ & 12 & 11 & 2 & 0 & 0 \\
& $1-9$ & 0 & 1 & 4 & 4 & 2 \\
& $<1$ & 0 & 0 & 6 & 8 & 10 \\
\hline
\end{tabular}




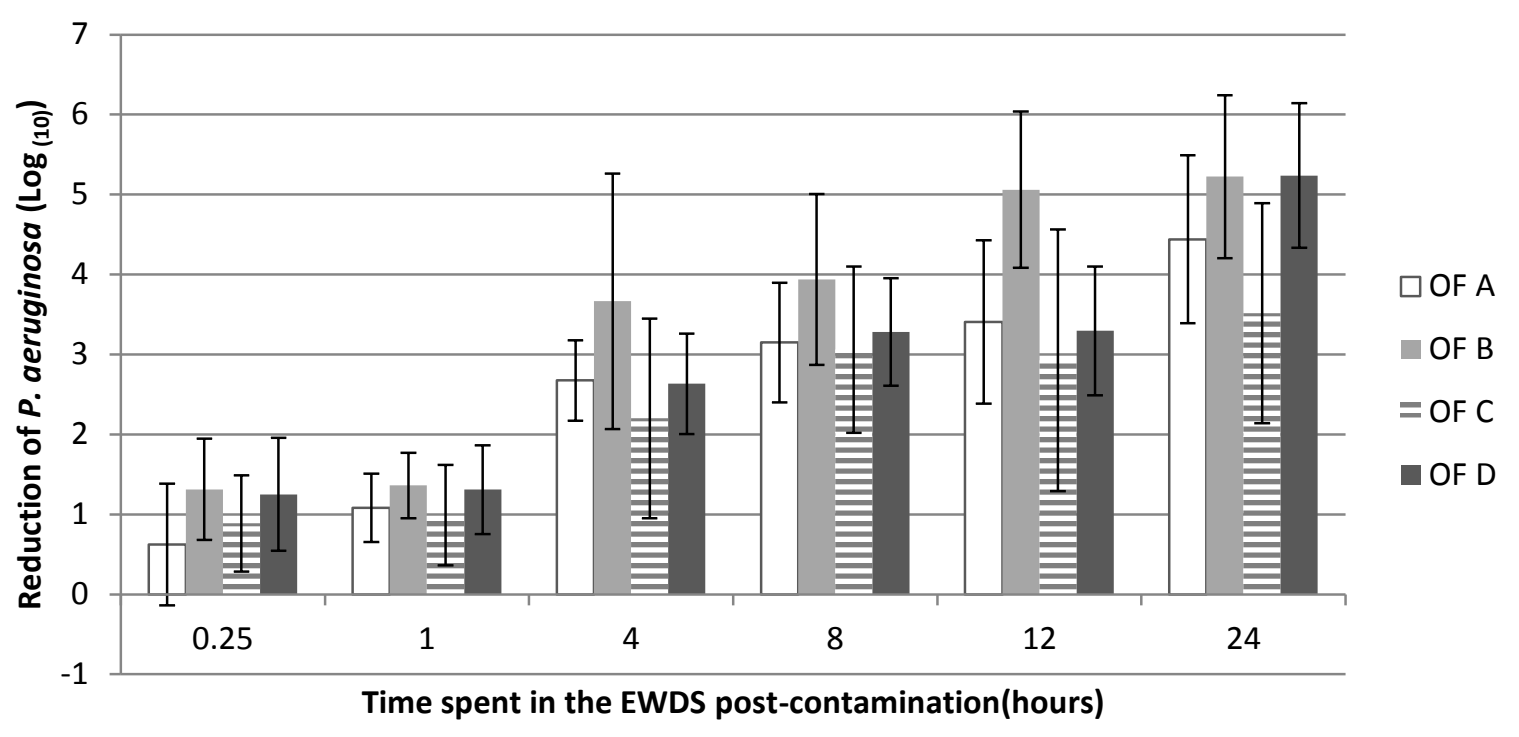

Figure 1. Reduction $\left(\log _{(10)}\right)$ in cloth-transferred $P$. aeruginosa on outlet fitting types [mean $(n=12) \pm$ standard deviation] 